\title{
Statistical Face Models for the Prediction of Soft-Tissue Deformations After Orthognathic Osteotomies
}

\author{
Sebastian Meller ${ }^{1}$, Emeka Nkenke ${ }^{2}$, and Willi A. Kalender ${ }^{1}$ \\ 1 Institute of Medical Physics, University of Erlangen-Nürnberg, Germany \\ sebastian.meller@imp.uni-erlangen.de \\ 2 Department of Oral and Maxillofacial Surgery, \\ University of Erlangen-Nürnberg, Germany
}

\begin{abstract}
This paper describes a technique to approximately predict the facial morphology after standardized orthognathic ostoetomies. The technique only relies on the outer facial morphology represented as a set of surface points and does not require computed tomography (CT) images as input. Surface points may either be taken from 3D surface scans or from 3D positions palpated on the face using a tracking system. The method is based on a statistical model generated from a set of pre- and postoperative 3D surface scans of patients that underwent the same standardized surgery. The model contains both the variability of preoperative facial morphologies and the corresponding postoperative deformations. After fitting the preoperative part to 3D data from a new patient the preoperative face is approximated by the model and the prediction of the postoperative morphology can be extracted at the same time. We built a model based on a set of 15 patient data sets and tested the predictive power in leave-one-out tests for a set of relevant cephalometric landmarks. The average prediction error was found to be between 0.3 and $1.2 \mathrm{~mm}$ at all important facial landmarks in the relevant areas of upper and lower jaw. Thus the technique provides an easy and powerful way of prediction which avoids time, cost and radiation required by other prediction techniques such as those based on CT scans.
\end{abstract}

\section{Introduction}

In oral and maxillofacial surgery one of the major issues is the correction of dentoskeletal deformities of the skull. The standard type of orthognathic surgery is to advance the upper jaw and to push back the lower jaw. Its aim is to establish a normal masticatory function along with an improvement in facial esthetics 1. To obtain true informed consent of the patient in orthognathic surgery, the surgeon must effectively explain possible treatment outcomes to the patient. Unfortunately, up to now the few existing methods to predict the facial appearance changes that will result from orthognathic surgery are not reliable and require 3D imaging [2]. Computed tomography (CT) scans are often used as basic data for the prediction of the postoperative facial surface. Most 


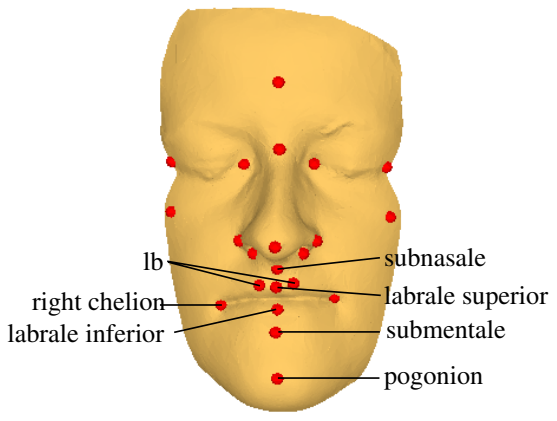

(a)

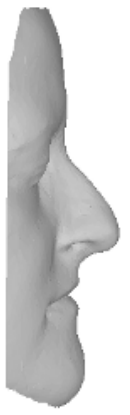

(b)

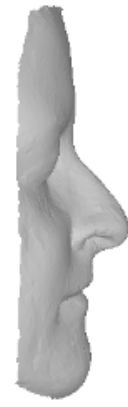

(c)

Fig. 1. Example for a typical input data set: a preoperative face acquired from an optical 3D sensor ( $a$ and b) with manually marked anatomical landmarks (a) and the postoperative face one year after surgery (c) in which the upper jaw has been moved forward and the lower jaw backwards.

of these methods try to model the soft tissue deformation with the aid of finite element or spring models based on the planned surgical change of the underlying bone structure. To date, there is little information available on the accuracy of these predictions 345. Commercial applications exist that allow geometric deformations of photographs or scanned 3D models in order to plan the surgical outcome but there is no quantitative validation on how realistic these predictions are. It has been the aim of this study to introduce a new prediction approach avoiding time, cost and radiation introduced by the CT scan and to evaluate the accuracy of this approach. The presented method is based on a 3D statistical model built from a set of 3D surface scans of different patients before and 12 months after the same type of orthognathic surgery (see Fig. 11). The variety of preoperative facial morphologies as well as the corresponding postoperative deformations are both captured in the model. The model can be fitted to the face of a new patient whose facial morphology is partly known as a set of 3D positions on the face which can either be digitally acquired by 3D surface scans or from positions directly palpated using a tracking system. After this fitting process the prediction can simply be extracted from the model.

We will present our statistical models in section 2 and how the model was built from the given data in section 3. The model fitting algorithm is described in section 4 , followed by an evaluation in section 5 .

\section{Extended Shape Models}

Cootes and Taylor first presented the idea of deformable statistical models [6] for the purpose of image segmentation in 1992. The main assumption is that a class of shapes can be described by a relatively small number of linear variations of the average shape. Object shapes are described in a compact way as a vector of 
boundary point coordinates. A class of object shapes is modeled by an average shape and a linear combination of vectors that describe the possible variations of the shape compared to the average shape as deformation vectors. The model can be easily deformed by changing the weights of the deformation vectors within a limiting range keeping the shape always plausible, i.e. inside the given class of shapes.

Technically the shape of an object is described as a point set $\mathcal{F}_{i}$ of $S$ points $\mathbf{p}_{i j}, j=1 \ldots S$ on the object surface, given $N$ objects $(i=1 \ldots N)$, in this case preoperative facial morphologies from different patients. Assuming at this point that a dense correspondence map between all shapes has already been defined and all shapes are already aligned in space, then we can describe each input shape as a vector $\mathbf{x}_{i}$ by concatenating the $\mathrm{x}-, \mathrm{y}$ - and $\mathrm{z}$-coordinates of the points, yielding a vector of dimension $M=3 S$. By applying an eigenvalue decomposition [7] to the covariance matrix of these vectors we get a number of eigenvalues $\lambda_{i}$ with associated orthogonal eigenvectors $\mathbf{v}_{i}$. It can be shown that there are at most $N-1$ eigenvalues with $\lambda_{k}>0$, if $N<M$ [6]. By defining a matrix $\mathbf{V}$ from the eigenvectors $\mathbf{v}_{k}$ associated to these eigenvalues, each of the input shape vectors $\mathbf{x}_{i}$ as well as arbitrary anatomically plausible shapes $\mathbf{x}$ can be described as a sum of the average shape vector $\overline{\mathbf{x}}$ plus a linear combination of these eigenvectors with a weight vector $\mathbf{b}$ :

$$
\begin{aligned}
& \mathbf{x}=\overline{\mathbf{x}}+\mathbf{V b}, \mathbf{V}=\left(\mathbf{v}_{1}, \mathbf{v}_{2}, \ldots, \mathbf{v}_{N-1}\right), \\
& -3 \sqrt{\lambda_{k}}<b_{k}<3 \sqrt{\lambda_{k}}, k=1 \ldots N-1
\end{aligned}
$$

Since the eigenvalues are equal to the variance within the input data in direction of the associated eigenvector, we can assume plausible shapes generated by a linear combination of weights that are constrained to a variation of three standard deviations per eigenvector [6].

In order to include the transition between pre- and postoperative morphology into the model, we extended this original approach by adding the displacement vector field from pre- to postoperative facial morphology as a second part of the input vector: We assume at this point that preoperative and postoperative surfaces have already been registered and that a dense correspondence between them has been established. In other words, for each preoperative face point $\mathbf{p}_{i j}$ of a patient $\mathcal{F}_{i}$ there is a corresponding postoperative point $\mathbf{q}_{i j}$. The displacement vector field between pre- and postoperative face can thus be defined as $\mathbf{d}_{i j}=$ $\mathbf{q}_{i j}-\mathbf{p}_{i j}$. The definition of the vector $\mathbf{x}_{i}$ is extended to contain the coordinates $\mathbf{p}_{i j}$ first and then the displacement vector field $\mathbf{d}_{i j}$, thus doubling the dimension $M$ of the vector to $M=6 S$.

\section{Model Generation}

\subsection{Data Acquisition}

3D surface scans of 8 female and 7 male patients were acquired with an optical 3D sensor (SCAN3D, 3D-Shape GmbH, Erlangen, Germany), both preoperatively and one year after the surgery in which the upper jaw was moved forward 
and the lower jaw backwards, both between 3 and $7 \mathrm{~mm}$ (e.g. see Fig. 1). The postoperative scans were first rigidly registered to the preoperative scans as described by Maier et al. [8]. On all pre- and postoperative faces several important cephalometric landmarks were manually identified (see Fig. 1(a)).

\subsection{Correspondence Mapping}

A precondition for the statistical modeling is a dense correspondence between all input shapes. A manual correspondence mapping which involves marking mutually corresponding points on all input data sets is only feasible for a small subset of anatomical landmarks. Therefore several correspondence mapping algorithms have been proposed in the past 91011. We applied our own approach 12 that ensures smooth and dense correspondence mapping. It uses only moderate manual interaction and is based on the multiple deformation of a template surface to each of the input surfaces. The necessary non-rigid template-to-target registrations are performed in three steps: first, an affine registration based on the set of marked landmarks on both meshes [13] is performed, followed by second, a 3D thin plate spline deformation [14] which brings the template to exact alignment with the target mesh at the landmark positions. Thirdly, for refinement, a non-rigid iterative morphing approach is applied. It is based on four kinds of springs attached to the vertices of the template triangle mesh that determine the deformation of the template surface. Two of the springs minimize the surface distance to the target surface while the other two maintain the overall shape of the template surface by minimizing changes in inter-vertex edge lengths and intertriangle edge angles compared to the original template surface. In each iteration for each of the template vertices a deformation vector is calculated composed of the four spring forces where each type of spring is weighted differently.

We applied the same technique for the inter-patient registration of a preoperative template face to each of the other faces and for the intra-patient registrations of each pair of pre- and postoperative facial morphologies. As a result all pre- and postoperative faces are described with the same mesh topology with mutually corresponding vertices.

\subsection{Alignment and Model Calculation}

In order to compensate for translation and rotation differences in the given data sets we applied an iterative Procrustes alignment [15], again based on the manually marked landmark sets for each patient. At this point the model is generated as described in section 2, including the displacement vector field to the postoperative facial morphology into the shape vector.

\section{Model Fitting and Prediction}

The preoperative part of the generated model can be fitted to given data from an arbitrary face. We assume that the data is given as a $3 \mathrm{D}$ set of points on the unknown preoperative face. These may either be single point positions at known 
anatomical landmarks or whole point clouds on the face. The fitting process thus involves the determination of a rigid transformation $\mathbf{T}_{\mathrm{fit}}$ that transforms the model coordinates into the target coordinate system and the shape parameter vector $\mathbf{b}_{\text {fit }}$ that deforms the model surface such that it optimally fits the given point set. After the model-based deformation a further non-linear deformation can be applied (section 4.2).

\subsection{Model Fitting}

We partly applied the fitting algorithm described by Blanz et al. 16. The method uses a linear matrix that maps the given vector $\mathbf{x}$ of model coordinates onto a subset of points and applies a linear transformation, in our case a rigid transformation that registers the model with the patient. Assuming the correspondences from given points to model vertices are known, the shape parameter vector $\mathbf{b}$ which generates the model face (according to (10) that best fits the given points can be found in a closed solution by solving a linear equation system after applying a singular value decomposition [7].

In this study we only used the set of predefined landmarks for model fitting for which the corresponding vertices on the model surface are known. The approach can be extended to include the whole preoperative facial morphology by starting with an initial model fit defined by known landmarks. Correspondences for the remaining vertices can then be acquired by projection onto the already fitted model surface. Thus, the fitting can be iteratively refined.

\subsection{Refinement Deformation}

In order to perfectly fit the model surface to the given point set and consider shape variances not captured in the statistical model, a non-linear deformation can follow the fitting process described above. In this step a thin-plate spline warping is applied to the fitted model face based on the known pairs of corresponding landmarks between model and patient. In this way the preoperative model face perfectly fits the patient face at the landmark positions.

\subsection{Face Prediction}

The above fitting process defines a shape parameter vector $\mathbf{b}$ and therefore a vector $\mathbf{x}$ that represents in its first half an approximation of the given preoperative face. Since the displacement vector field to the postoperative face is stored in the second part of the same vector (see section 2) and is therefore determined by the same shape parameter vector according to (1), a prediction of the postoperative facial morphology can now be acquired by adding this adapted displacement vector field to the fitted preoperative face.

\section{$5 \quad$ Evaluation Results}

The evaluation was carried out in 15 leave-one-out tests. In each test one of the 15 data sets was defined as the test data set; the model was generated from the 


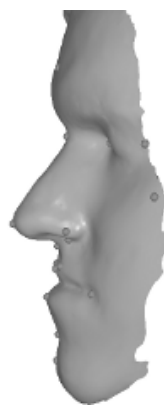

(a)

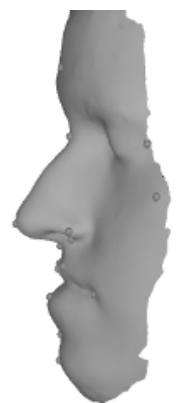

(b)

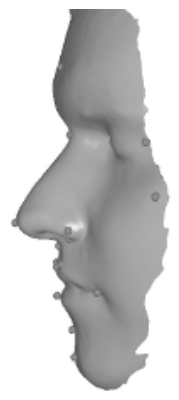

(c)

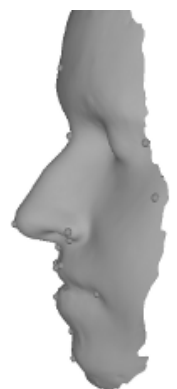

(d)

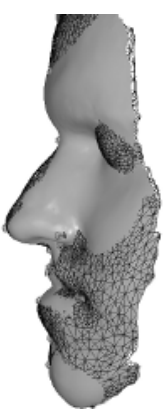

(e)

Fig. 2. Example for a prediction: A preoperative face of a patient (a) was landmarked and the statistical model generated without this patient was fitted to these landmarks as can be seen in (b). The actual postoperative outcome (c) one year after surgery can then be compared with the prediction $(\mathrm{d})$ produced by the model, both combined in one image in (e).

14 remaining data sets and then tested against the test model (see example in Fig. 2).

We used the manually marked anatomical landmarks on the test data set in order to fit the model. The predicted postoperative face could then be compared to the known postoperative face. We measured the prediction error at a subset of the marked cephalometric landmarks:

- subnasale: point at the tip of the angle formed by colunella and upper lip

- labrale superior: most anterior point of the upper lip

- lb left and right: top of the cupid's bow of the upper lip

- chelion left and right: points located at the labial commissure

- labrale inferior: most anterior point of the lower lip

Table 1. Statistical evaluation of the average absolute prediction error in $\mathrm{mm}$ at certain anatomical landmarks from leave-one-out tests in 15 cases, compared to the maximum displacement caused by the surgeries at these landmarks.

\begin{tabular}{|l|l|l|l|l|}
\hline Landmark & Mean & SD & Max & $\begin{array}{l}\text { Max. } \\
\text { actual }\end{array}$ \\
\hline pogonion & 1.2 & 1.2 & 3.8 & 8.5 \\
\hline submentale & 1.0 & 1.1 & 3.4 & 7.5 \\
\hline labrale inferior & 1.0 & 1.1 & 3.5 & 5.8 \\
\hline labrale superior & 0.6 & 0.6 & 2.7 & 3.1 \\
\hline lb left & 0.5 & 0.5 & 2.1 & 2.5 \\
\hline lb right & 0.7 & 0.8 & 3.6 & 4.1 \\
\hline chelion left & 0.8 & 0.5 & 1.9 & 2.6 \\
\hline chelion right & 0.8 & 1.0 & 3.6 & 3.2 \\
\hline subnasale & 0.3 & 0.4 & 1.6 & 2.3 \\
\hline
\end{tabular}


- submentale: most posterior point between lower lip and chin

- pogonion: most anterior point of the chin

Results are shown in Table 1, The measured average prediction errors over all 15 cases at the landmarks with the greatest deviations (pogonion, submentale and labrale inferior) were about $1.2 \mathrm{~mm} \pm 1.2 \mathrm{~mm}$ with rare outliers below $4 \mathrm{~mm}$ whereas the absolute displacements ranged from $2.3 \mathrm{~mm}$ to $8.5 \mathrm{~mm}$. All other landmark positions showed average prediction errors below $1 \mathrm{~mm}$.

\section{Conclusions and Discussion}

We showed how statistical face models that include the shape change between pre- and postoperative facial morphology can be successfully applied to predict the postoperative facial morphology after orthognathic surgery from sparse 3D data of a patient's preoperative face. Although sophisticated nonlinear finite element modeling may be an appropriate alternative, there is no proof from the current literature that these techniques generate valid predictions of the human face. The presented method avoids time, cost and radiation associated with other techniques that require a CT scan. It is fast and simple during application. The fit and prediction is calculated within a few seconds after minimal manual interaction. Even based on a relatively small data base of 15 male and female cases, the comparison between the real postoperative outcome of the facial surfaces and the predicted surfaces revealed that the average differences were below 1.5 $\mathrm{mm}$ for landmarks placed on the relevant areas of upper and lower jaw. This new type of prediction of the outcome of orthognathic surgery is favorable for several reasons: differences between facial surfaces below $1.5 \mathrm{~mm}$ cannot be easily distinguished by the normal observer and the thickness of the facial soft tissue already changes during the course of the day in this range. In addition, the facial surface is influenced by changes of body weight and aging. Therefore, it will be difficult to achieve a better prediction accuracy than the one shown in this study. Of course, the application of the method is restricted to the kind of surgery and the variability of facial morphologies covered in the data base. Thus, future work will include increasing the data base to more patient data sets from different types of surgeries, different ethnic groups and ages. The new method may also find its application in plastic facial surgery where well standardized procedures allow an accurate prediction by the proposed technique.

\section{References}

1. Xia, J., Ip, H., Samman, N., Wong, H., Gateno, J., Wang, D., Yeung, R., Kot, C., Tideman, H.: Three-dimensional virtual-reality surgical planning and soft-tissue prediction for orthognathic surgery. IEEE Trans Inf Technol Biomed 5 (2001) 97-107

2. Nkenke, E., Langer, A., Laboureux, X., Benz, M., Maier, T., Kramer, M., Häusler, G., Kessler, P., Wiltfang, J., Neukam, F.: Validation of in vivo assessment of facial soft-tissue volume changes and clinical application in midfacial distraction: a technical report. Plast Reconstr Surg 112 (2003) 367-80 
3. Koch, R.M., Roth, S.H.M., Gross, M.H., Zimmermann, A.P., Sailer, H.F.: A framework for facial surgery simulation. In: SCCG '02: Proceedings of the 18th Spring Conference on Computer Graphics, ACM Press (2002) 33-42

4. Gladilin, E., Zachow, S., Deuflhard, P., Hege, H.C.: On constitutive modeling of soft tissue for the long-term prediction of cranio-maxillofacial surgery outcome. In Lemke, H.U., Vannier, M.W., Inamura, K., Farman, A.G., Doi, K., Reiber, J.H.C., eds.: Proc. CARS. Volume 1256 of ICS., London, Elsevier (2003) 3438

5. Westermark, A., Zachow, S., Eppley, B.: Three-dimensional osteotomy planning in maxillofacial surgery including soft tissue prediction. Journal of Craniofacial Surgery 16 (2005) 100-104

6. Cootes, T.F., Taylor, C.J., Cooper, D.H., Graham, J.: Training models of shape from sets of examples. In: Proc. BMVC, Berlin, Springer (1992) 266-275

7. Press, W., Flannery, B., Teukolsky, S., Vetterling, W.: Numerical Recipes: The Art of Scientific Computing. 2nd edn. Cambridge University Press, Cambridge (UK) and New York (1992)

8. Maier, T., Benz, M., Schön, N., Nkenke, E., Neukam, F., Vogt, F., Häusler, G.: Automatic coarse registration of 3D surface data in oral and maxillofacial surgery. In Buzug, T., Lueth, T., eds.: Perspective in image-guided surgery, Singapore, World Scientific (2004) 51-58

9. Hutton, T.J., Buxton, B.F., Hammond, P.: Dense surface point distribution models of the human face. In Staib, L., ed.: IEEE Workshop on Mathematical Methods in Biomedical Image Analysis. (2001) 153-160

10. Lamecker, H., Seebass, M., Hege, H.C., Deuflhard, P.: A 3D statistical shape model of the pelvic bone for segmentation. In Fitzpatrick, J., Sonka, M., eds.: Proceedings of SPIE Medical Imaging 2004: Image Processing. Volume 5370. (2004) 1341-1351

11. Fleute, M., Lavallée, S.: Building a complete surface model from sparse data using statistical shape models: Application to computer assisted knee surgery system. In: MICCAI. Volume 1496 of LNCS., Springer-Verlag (1998) 879-887

12. Meller, S., Kalender, W.: Building a statistical shape model of the pelvis. In Lemke, H.U., ed.: Computer Assisted Radiology and Surgery (CARS), Chicago, IL (2004)

13. Umeyama, S.: Least-squares estimation of transformation parameters between two point patterns. IEEE Trans. PAMI 13 (1991) 376-380

14. Bookstein, F.L.: Principal warps: Thin-plate splines and the decomposition of deformations. IEEE Trans. PAMI 11 (1989) 567-585

15. Goodall, C.: Procrustes methods in the statistical analysis of shape. Journal of the Royal Statistical Society 53 (1991) 285-339

16. Blanz, V., Mehl, A., Vetter, T., Seidel, H.P.: A statistical method for robust 3D surface reconstruction from sparse data. In: International Symposium on 3D Data Processing, Visualization and Transmission, Thessaloniki, Greece (2004) 